\title{
Síndrome de dolor abdominal mediado centralmente
}

\author{
Centrally mediated abdominal pain syndrome \\ José A. Velarde-Ruiz Velasco y Diana K. Tapia-Calderón \\ Servicio de Gastroenterología, Hospital Civil de Guadalajara Fray Antonio Alcalde, Guadalajara, México
}

\section{RESUMEN}

El síndrome de dolor abdominal mediado centralmente es una patología que cursa con dolor abdominal frecuente y continuo, cuya fisiopatología es bastante desconocida. El manejo de estos pacientes es muy complejo, tanto por el tipo de dolor como por sus características psicológicas y conductuales, por lo que es necesario un diagnóstico acertado y un enfoque multidisciplinario. El éxito del tratamiento se basará en la empatía que el médico sea capaz de generar con el paciente y en la educación del paciente sobre su enfermedad, aplicándose medidas farmacológicas y psicológicas.

Palabras clave: Síndrome de dolor abdominal mediado centralmente. Medidas psicológicas. Medidas conductuales. Empatía.

\section{ABSTRACT}

Centrally-mediated abdominal pain syndrome is a pathology that presents with frequent and continuous abdominal pain, and its pathophysiology is rather unknown. The management of these patients is very complex, both due to the type of pain and its psychological and behavioral characteristics, which is why an accurate diagnosis and a multidisciplinary approach are necessary. The success of the treatment will be based on the empathy that the doctor is able to generate towards the patient and on the patient's education about the disease, applying pharmacological and psychological measures.

Key words: Centrally mediated abdominal pain syndrome. Psychological measures. Behavioral measures. Empathy.

\section{Correspondencia:}

José A. Velarde-Ruiz Velasco

E-mail: velardemd@yahoo.com.mx
NeuroGastroLatam Rev. (ahead of print)

Recibido: 16-03-2020

Aceptado: 29-06-2021

DOI: 10.24875/NGL.20000007

2462-7011/@ 2021 Sociedad Latinoamericana de Neurogastroenterología, A.C. Published by Permanyer. This is an open access article under the CC BY-NC-ND license (http://creativecommons.org/licenses/by-nc-nd/4.0/). 


\section{INTRODUCCIÓN}

Los trastornos funcionales gastrointestinales (TFGI) son alteraciones del eje intestino-cerebro que suceden en ausencia de patología gastrointestinal alguna. El conocimiento reciente de los múltiples factores fisiopatológicos involucrados en la generación de estos trastornos ha llevado a la modificación de la definición de los TFGI, ahora conocidos como trastornos de la interacción intestino-cerebro (TIIC) ${ }^{1,2}$. Hasta el 35\% de la población general de EE.UU., Canadá y Reino Unido cumplen los criterios de Roma IV para TIIC. Comparados con controles sanos, los pacientes con TIIC presentan mayor somatización, peor calidad de vida e incremento en las intervenciones médicas y quirúrgicas ${ }^{3}$.

De acuerdo con la última clasificación Roma IV, el «síndrome de dolor abdominal mediado centralmente» (SDAMC), antes conocido como «síndrome de dolor abdominal funcional», y el «síndrome del intestino narcótico» (SIN) o «hiperalgesia gastrointestinal inducida por opiáceos», forman parte de los «trastornos gastrointestinales mediados centralmente ${ }^{2}$.

\section{SÍNDROME DE DOLOR ABDOMINAL MEDIADO CENTRALMENTE}

El SDAMC se caracteriza por dolor abdominal constante e intenso con episodios de exacerbación de los síntomas, con inicio en los últimos seis meses, representando para el paciente una importante pérdida de la actividad de la vida diaria y la calidad de vida ${ }^{4}$.

La frecuencia de presentación del SDAMC es menor que la de otros TIIC, como el síndrome de intestino irritable (SII) y la dispepsia funcional (DF) entre otros, con una prevalencia reportada que varía entre 0.5 y el $2.1 \%$. Es dos veces más frecuente en pacientes del sexo femenino, presentando su máxima prevalencia en la cuarta década de la vida para luego ir disminuyendo con la edad ${ }^{5}$. En una reciente encuesta realizada en 5,931 adultos de países de habla inglesa, se reportó que hasta 2,083 (35.1\%) presentaban síntomas compatibles con TIIC; entre los cuales los trastornos intestinales fueron los más predominantes (28.1\%), y solo uno de los sujetos incluidos en el estudio cumplió criterios diagnósticos para SDAMC $(0.02 \%)^{3}$.

Los pacientes con SDAMC consultan frecuentemente a distintos especialistas y son sometidos a múltiples procedimientos endoscópicos y quirúrgicos innecesarios, sobrecargando los costos en salud. Además, de manera similar a la del SII, tienen una elevada tasa de ausentismo laboral ${ }^{4}$. Kilgallon, et al. reportaron que hasta el 68\% de los pacientes con dolor abdominal crónico habían sido sometidos a cirugía abdominal previa, la mitad de ellos con diagnóstico de SDAMC. Las cirugías más frecuentemente realizadas fueron apendicectomía, colecistectomía e histerectomía, la cirugía no resolvió la sintomatología en ningún paciente e incluso el 35\% reportaron empeoramiento de sus síntomas con ella ${ }^{6}$.

\section{FISIOPATOLOGÍA DEL SÍNDROME DE DOLOR ABDOMINAL MEDIADO CENTRALMENTE}

La fisiopatología del SDAMC es similar a la de otros trastornos que cursan con dolor crónico como el SII y la DF. Los pacientes con SII presentan hipersensibilidad e hipervigilancia 
al dolor secundaria a un aumento de la señalización aferente del dolor visceral relacionada con los alimentos, experiencias tempranas de la vida, inflamación de la mucosa intestinal, la menstruación, el antecedente de cirugía abdominal o infecciones gastrointestinales. En contraste, en los pacientes con SDAMC existe poca o nula asociación con la alimentación o la defecación ${ }^{4,5}$.

La hipersensibilidad visceral ha sido involucrada en la fisiopatología de los TIIC, contribuyendo con algunos síntomas clave en este grupo de pacientes, sin embargo, no todos los pacientes presentan hipersensibilidad visceral. Un estudio reciente demostró que existe un aumento en la gravedad de los síntomas gastrointestinales asociado con un aumento en la sensibilidad gastrointestinal en pacientes con SII y $\mathrm{DF}^{7}$. En contraste, los pacientes con SDAMC tienen un umbral de percepción a la distensión rectal normal, a diferencia de los pacientes con SII con estreñimiento (SII-E), que tienen un umbral a la distensión rectal disminuida ${ }^{8}$. El SDAMC se distingue de otros TIIC por su fuerte componente central, resulta de la sensibilización central y desinhibición de las señales del dolor, más que del aumento de la excitabilidad periférica aferente ${ }^{2}$.

Como ocurre con otros trastornos de dolor somático, es difícil incluir este trastorno en las categorías tradicionales de dolor neuropático $^{5}$. Fisiológicamente, el cerebro recibe información interoceptiva de las vísceras abdominales, que se combina con información cognitiva, emocional y sensorial para su interpretación consciente en la ínsula anterior. Los estímulos nocivos activan los receptores del tracto gastrointestinal que son sensibles a estímulos químicos, mecánicos o inflamatorios. Esta señal se transmite a la corteza somatosensorial primaria y secundaria a través del tracto espinotalámico y al tálamo medial, corteza cingulada anterior e ínsula a través de los tractos espinorreticular y espinomesencefálico, áreas que participan en los aspectos afectivos-motivacionales del dolor visceral ${ }^{9}$. La disfunción del neuroeje que conduce al dolor abdominal funcional podría ser consecuencia del aumento de la señalización aferente del dolor visceral, sensibilización central de la asta dorsal, alteraciones en la modulación descendente o en la amplificación central por una pérdida en los filtros del dolor?.

\section{CLÍNICA DEL SÍNDROME DE DOLOR ABDOMINAL MEDIADO CENTRALMENTE}

La característica principal del SDAMC es el dolor abdominal crónico y constante, de al menos tres meses de duración con inicio de los síntomas seis meses antes. Se trata de un dolor de tipo cólico, en ocasiones quemante, prolongado y sin una localización bien definida. Frecuentemente los pacientes refieren síntomas extraintestinales, como dolor musculoesquelético ${ }^{5,6}$. Puede existir asociación con otras condiciones de dolor crónico, como fibromialgia ${ }^{10}$.

Solo en el 34\% de los casos es posible identificar un factor desencadenante, que suele ser una cirugía abdominal previa o un cuadro de gastroenteritis infecciosa, en el resto no existe un factor desencadenante evidente ${ }^{6}$. Algunos pacientes también indican un hábito intestinal anormal, aunque no lo asocian al dolor constante ${ }^{5}$. 
Aunque no existe un perfil psicológico determinado en los pacientes con SDAMC, muchos de ellos pueden cumplir criterios de depresión, ansiedad y somatización. También son frecuentes los antecedentes de pérdidas no resueltas e incluso de abuso físico o se$\mathrm{xual}^{5}$. La mayoría de los pacientes negarán el papel de los factores psicosociales en el cuadro de dolor. Así, las características psicosociales del paciente y los patrones previos de búsqueda de atención médica, aunque no son criterios diagnósticos, pueden contribuir al diagnóstico 9 .

\section{Modelo miedo-evitación del dolor}

El paso de dolor agudo a crónico está influido por las condiciones fisiopatológicas del trastorno y por los factores psicosociales del paciente. De esta forma, algunos pacientes confieren al dolor un significado catastrófico, lo que provocará miedo al dolor y en consecuencia una conducta de evitación ${ }^{11}$.

El dolor catastrofista se caracteriza por la magnificación del valor de la amenaza del dolor, impotencia en el contexto del dolor y la incapacidad para inhibir adecuadamente los pensamientos relacionados con el dolor antes, durante o después de un encuentro doloroso, lo cual conlleva la evitación de situaciones en las que pueda ocurrir el dolor. El dolor catastrofista se ha asociado a menor calidad de vida, aumento del riesgo de uso inadecuado de opioides, interacciones sociales negativas y afectación de la relación médico-paciente ${ }^{12}$.

Estos pacientes, además, presentarán rasgos conductuales de inflexibilidad psicológica que provocará su obsesión en encontrar un tratamiento para su dolor, buscando el reconocimiento de su enfermedad ${ }^{12}$.

\section{SÍNDROME DEL INTESTINO NARCÓTICO 0 HIPERALGESIA GASTROINTESTINAL INDUCIDA POR OPIÁCEOS}

Se define como un aumento paradójico en el dolor abdominal asociado con uso continuo o el aumento de la dosis de los opioides. Tiene una prevalencia estimada del 5\% en los consumidores crónicos de opioides. El dolor abdominal es el síntoma principal, aunque puede acompañarse de náuseas, distensión abdominal, vómitos intermitentes y estreñimiento ${ }^{5,13}$.

En pacientes con dolor abdominal, los opioides pueden parecer útiles, sin embargo, la taquifilaxis ocurre de manera temprana, resultando en aumento de la dosis con o sin prescripción médica, esto conlleva a un aumento de los síntomas y reducción de la motilidad intestinal ${ }^{13}$. En el SIN, el dolor abdominal empeora cuando se disminuye la dosis del narcótico y mejora cuando el narcótico es reiniciado, existe una progresión de la frecuencia, duración e intensidad de los episodios de dolor y la sintomatología debe estar presente al menos tres meses en los últimos seis meses $^{5}$.

El tratamiento del SIN incluye el entendimiento y la modificación de la creencia del paciente de que los narcóticos le ayudan a controlar el dolor, la destoxificación de opiáceos tiene una tasa de éxito del $89.7 \%$. 


\section{EXPLORACIÓN FÍSICA DE LOS TRASTORNOS DE DOLOR ABDOMINAL MEDIADOS CENTRALMENTE}

Por definición, el examen físico de un paciente con SDAMC suele ser normal. Sin embargo, vale la pena analizar la presencia de cicatrices abdominales relacionadas con cirugías o con pruebas previas como factores iniciadores ${ }^{9}$. En la exploración puede ser útil la búsqueda activa del signo de Carnett. Este consiste en la presencia de un foco doloroso a la palpación del costado del músculo recto abdominal antes y después de que el paciente tense la pared abdominal de forma intencionada al levantar la cabeza o las piernas. La prueba es positiva si aparece dolor y sugiere causa musculoesquelética o atrapamiento del nervio cutáneo anterior, aunque en algunos pacientes con SDAMC puede positivizarse el signo de Carnett debido a la hipersensibilidad central y a la hipervigilancia, ${ }^{9,10}$.

Para distinguir entre dolor abdominal agudo y crónico, también puede ser útil en la exploración física el «signo de los ojos cerrados». En el dolor agudo, al realizar la palpación del abdomen, el paciente tendrá los ojos abiertos siempre, mientras que los pacientes con SDAMC tienen los ojos cerrados y hacen una mueca al momento de palpar el abdomen ${ }^{4}$. En ocasiones puede observarse eritema secundario a la aplicación de calor local en el abdomen y alodinia cutánea al tacto superficial de la piel abdominal ${ }^{10}$.

\section{PRUEBAS COMPLEMENTARIAS}

Solo se deben realizar exámenes complementarios en pacientes con signos de alarma o sospecha de alguna patología orgánica, ya sea estudio de sangre oculta en heces para descartar hemorragia gastrointestinal, hemograma, perfil bioquímico y estudios endoscópicos en pacientes con antecedentes de cáncer gástrico, de colon o de enfermedad inflamatoria intestinal ${ }^{4,5}$.

La tomografía computarizada de abdomen y pelvis es útil para descartar neoplasias o lesiones ginecológicas, por lo que es prudente solicitarla si no se ha realizado previamente ${ }^{5}$. La figura 1 muestra un algoritmo de diagnóstico y tratamiento para el SDAMC.

\section{TRATAMIENTO DE LOS TRASTORNOS DE DOLOR ABDOMINAL MEDIADOS CENTRALMENTE}

El tratamiento deberá basarse en la severidad de los síntomas y el grado de discapacidad que provocan. Siempre que se considere necesario, se deberá remitir al paciente a un especialista en salud mental y realizar simultáneamente el tratamiento médico y psicológico. También puede ser necesario el tratamiento en centros especializados en dolor (evitando el tratamiento con analgésicos opioides $)^{5}$. El abordaje y manejo óptimo del paciente con SDAMC se resume en las «4 R»: reconocimiento, relación, reemplazo y reducción ${ }^{10}$.

\section{Reconocimiento}

Es importante realizar un diagnóstico claro y positivo del SDAMC con el fin de conseguir la confianza del paciente, su compromiso con el tratamiento y para evitar intervenciones innecesarias o perjudiciales ${ }^{10}$. 


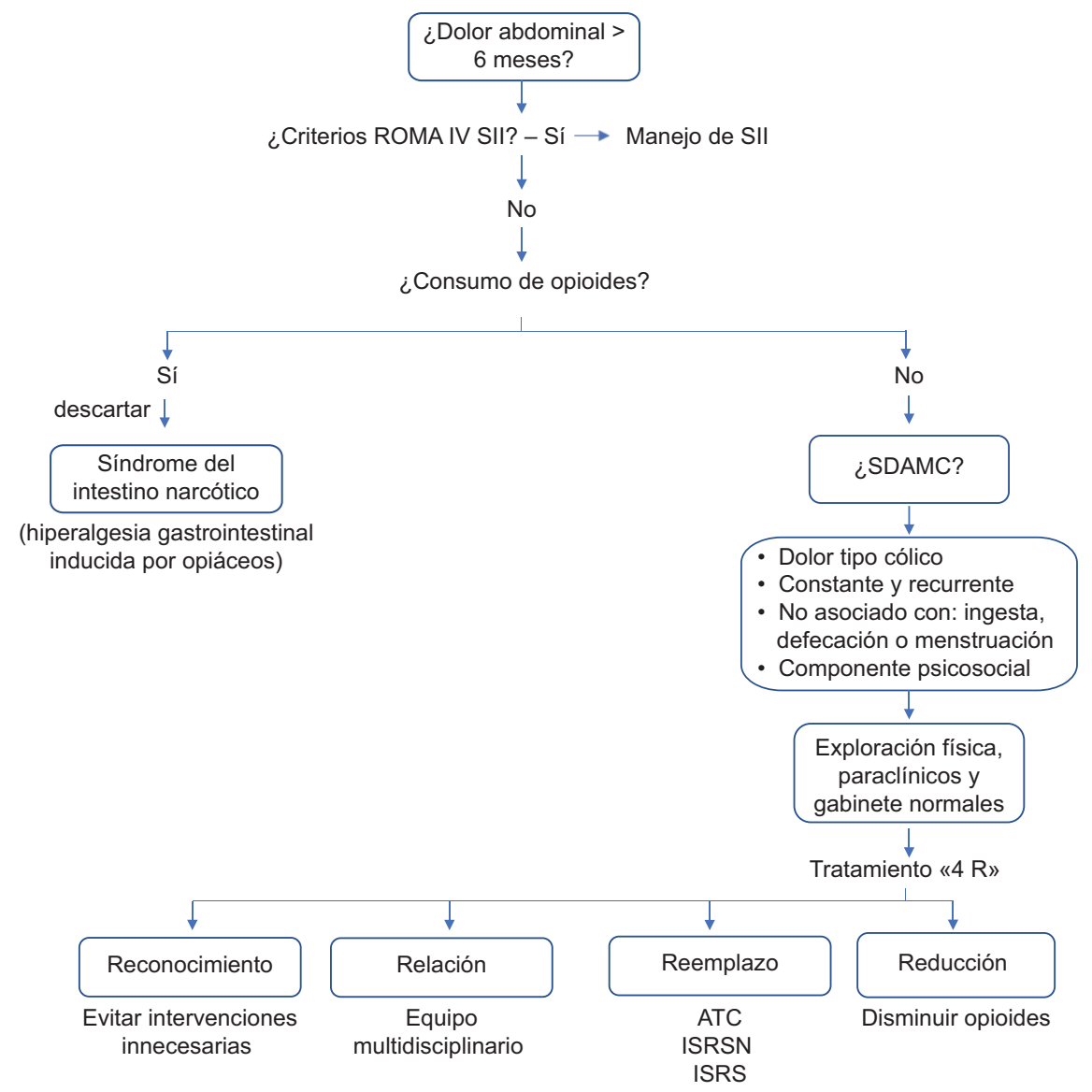

Figura 1. Algoritmo de diagnóstico y tratamiento para el síndrome de dolor abdominal mediado centralmente. ATC: antidepresivos tricíclicos; ISRSN: inhibidores selectivos de la recaptación de serotonina y noradrenalina; ISRS: inhibidores selectivos de la recaptación de serotonina.

\section{Relación}

Es fundamental una buena relación médicopaciente, ya que, al ser un problema crónico, probablemente el paciente habrá tenido experiencias negativas con otros profesionales. Puede ser beneficiosa la relación del paciente con un equipo multidisciplinario, en el que se debería incluir además del gastroenterólogo, un especialista en dolor, un fisioterapeuta y un psicólogo clínico. Para alcanzar una buena relación médico-paciente, el profesional de la salud deberá ser empático con el paciente, educarlo sobre su trastorno, así como establecer metas terapéuticas realistas ${ }^{10}$. De cualquier forma, la relación del médico tratante con otros profesionales de la salud es muy importante para evitar la realización de pruebas innecesarias ${ }^{12}$.

\section{Reemplazo}

El uso de neuromoduladores en el tratamiento de los TIIC puede ser útil para disminuir 
el estrés psicológico, la ansiedad, la hipervigilancia, la atención selectiva y la catastrofización del dolor, en especial en pacientes con enfermedades psiquiátricas asociadas como depresión mayor; además de la disminución del dolor, al regular a la baja la señalización visceral ${ }^{6}$.

El tratamiento farmacológico tiene una eficacia limitada. Se pueden utilizar antidepresivos tricíclicos (ADT), ligandos $\alpha 2 \delta$ (como gabapentina y pregabalina) e inhibidores selectivos de la recaptación de serotonina (ISRS) y los inhibidores de la recaptura de serotonina y noradrenalina $(\text { ISRSN })^{10}$. Sin embargo, ninguno de los tratamientos psicofarmacológicos ha sido aprobado por la Food and Drug Administration para manejo del dolor en TIIC, por lo que las recomendaciones actuales están basadas en ensayos clínicos, series de casos y opinión de expertos $^{6}$. Los neuromoduladores incluyen los siguientes:

- ADT: son los neuromoduladores más frecuentemente utilizados; son útiles en el tratamiento de SII-E, SDAMC, dolor torácico funcional, dolor anorrectal y DF. Son más efectivos que los ISRS en el manejo del dolor y sus propiedades anticolinérgicas son benéficas en pacientes con diarrea, la inhibición $\mathrm{H} 1$ disminuye el insomnio. Se deben administrar por la noche por sus propiedades sedantes ${ }^{6}$. Los efectos adversos más frecuentes son sedación o agitación, hipotensión, constipación e insomnio entre otros ${ }^{5}$.

Las aminas terciarias como la amitriptilina y la imipramina tienen mayores efectos adversos. Una dosis baja inicial de $10 \mathrm{mg}$ /día puede disminuir los efectos adversos; la dosis del ADT puede ajustarse en las primeras cuatro a seis semanas de tratamiento en el rango de 25 a $75 \mathrm{mg} /$ día por las noches, e incluso aumentar hasta $100 \mathrm{a} 150 \mathrm{mg}$ si hay adecuada tolerancia ${ }^{6,14}$. Debido al potencial arritmogénico de los ADT, se debe realizar un electrocardiograma basal y descartar prolongación del intervalo QT, bloqueo de rama izquierda o bifascicular, y evitar su uso en pacientes con antecedente de infarto agudo de miocardio $^{15}$.

- ISRS: el efecto analgésico de los ISRS es menor al de los ADT. Pueden utilizarse en pacientes con pobre control de los síntomas o en aquellos con ansiedad, depresión mayor, ansiedad social y fobias específicas o en pacientes con somatización o hipervigilancia. Los efectos adversos más frecuentemente reportados son náuseas, vómitos, disfunción sexual, diarrea, ansiedad y temblor 6 . La sertralina, el citalopram y el escitalopram son los que tienen menores interacciones farmacológicas gracias a su mínima interacción con el citocromo P4506.

- ISRSN:promueven la analgesiay sonútilesen el tratamiento de condiciones como la fibromialgia y el dolor neuropático. Su uso en el manejo del dolor visceral no ha sido adecuadamente estudiado, están indicados en pacientes con trastornos del dolor abdominal mediados centralmente que fracasan al tratamiento inicial con $\mathrm{ADT}^{6}$. Pueden ocasionar palpitaciones, sudoración, mareo, disfunción sexual, diarrea y pesadillas ${ }^{14,16}$. La ventaja de los ISRSN frente a los ADT es que no tienen el efecto antihistamínico o anticolinérgico. Por ejemplo, la duloxetina puede utilizarse a dosis de 30 a $90 \mathrm{mg} /$ día, mientras que la venlafaxina requiere dosis mínimas de $225 \mathrm{mg} /$ día. 
Los efectos adversos pueden reducirse al iniciar el tratamiento a dosis bajas ${ }^{14,15}$.

- Antipsicóticos atípicos: reducen la ansiedad y restablecen los patrones de sueño normales. Los efectos adversos más frecuentes son sedación y aumento de peso. La adición de quetiapina al tratamiento con ADT puede tener efectos positivos en el manejo de dolor gastrointestinal refractario ${ }^{5,15}$.

\section{Reducción de opioides}

En los TIIC el uso de opioides es inadecuado, sin embargo, en pacientes con dolor abdominal orgánico como cáncer los opioides pueden ser utilizados de forma temporal; en este grupo de pacientes es importante establecer metas de tratamiento e informar al paciente sobre el riesgo a corto y largo plazo con el uso de estos agentes ${ }^{13}$. Algunos factores de riesgo establecidos para el uso de opioides a largo plazo incluyen: sexo femenino, edad madura, bajo nivel socioeconómico, obesidad, múltiples comorbilidades y trastornos mentales previamente diagnosticados ${ }^{13}$.

Para poder realizar una correcta reducción de los opioides, se debe estabilizar la dosis que el paciente esté utilizando en el momento del tratamiento, utilizar formulaciones de duración prolongada y vías de administración que no sean inyectables ${ }^{10}$. Esta destoxificación debe ser realizada por expertos en esta materia. En algunos pacientes, la combinación de tratamientos puede ser útil. De esta forma, se puede utilizar conjuntamente antidepresivos con efecto ansiolítico junto a antipsicóticos atípicos o antidepresivos, simultáneamente al tratamiento psicológico ${ }^{4}$.

\section{CONCLUSIONES}

Los trastornos de dolor abdominal mediados centralmente son problemas crónicos que impactan negativamente sobre la calidad de vida de los pacientes que los padecen, por lo que es muy importante conocer las características del dolor y los síntomas que presenta el paciente. Para poder tratarlos adecuadamente es necesario una excelente relación médico-paciente, así como un enfoque multidisciplinario del paciente. Los neuromoduladores son el eje central del manejo tanto del SDAMC como del SIN, pero en este último, los programas de destoxificación son fundamentales.

\section{FINANCIAMIENTO}

No se recibió financiamiento para esta publicación.

\section{CONFLICTO DE INTERESES}

Los autores no tienen conflicto de interés en la realización de esta publicación.

\section{RESPONSABILIDADES ÉTICAS}

Protección de personas y animales. Los autores declaran que para esta investigación no se han realizado experimentos en seres humanos ni en animales.

Confidencialidad de los datos. Los autores declaran que han seguido los protocolos de su centro de trabajo sobre la publicación de datos de pacientes. 


\section{Derecho a la privacidad y consentimiento informado. Los autores declaran que en este artículo no aparecen datos de pacientes.}

\section{BIBLIOGRAFÍA}

1. Drossman DA, Hasler WL. Rome IV - Functional GI disorders: Disorders of gut-brain interaction. Gastroenterology. 2016;150(6):1257-61.

2. Tack J, Drossman DA. What's new in Rome IV? Neurogastroenterol Motil. 2017;29(9):151-63

3. Aziz I, Palsson OS, Törnblom H, Sperber AD, Whitehead WE, Simrén M. The prevalence and impact of overlapping Rome IV-diagnosed functional gastrointestinal disorders on somatization, quality of life, and healthcare utilization: A cross-sectional general population study in three countries. Am J Gastroenterol. 2018;113(1):86-96.

4. Sperber AD, Drossman DA. Review article: The functional abdominal pain syndrome. Aliment Pharmacol Ther. 2011;33(5):514-24.

5. Keefer L, Drossman DA, Guthrie E, Simrén M, Tillisch K, Olden K, et al. Centrally mediated disorders of gastrointestinal pain. Gastroenterology. 2016;150(6):1408-19.

6. Kilgallon E, Vasant DH, Green D, Shields PL, Hamdy S, Lal S, et al. Chronic continuous abdominal pain: evaluation of diagnostic features, iatrogenesis and drug treatments in a cohort of 103 patients. Aliment Pharmacol Ther. 2019;49(10):1282-92.
7. Simrén M, Törnblom H, Palsson OS, van Tilburg MAL, van Oudenhove L, Tack J, et al. Visceral hypersensitivity is associated with GI symptom severity in functional GI disorders: Consistent findings from five different patient cohorts. Gut. 2018;67(2):255-62.

8. Nozu T, Kudaira M. Altered rectal sensory response induced by balloon distention in patients with functional abdominal pain syndrome. Biopsychosoc Med. 2009;3(1):13.

9. Farmer AD, Aziz Q. Mechanisms and management of functional abdominal pain. J R Soc Med. 2014;107(9):347-54.

10. Paine P. Centrally mediated abdominal pain syndromes. Medicine [Internet]. 2019;47(6):354-7. Disponible en: https://doi.org/10.1016/j. mpmed.2019.03.003

11. Esteve R, Ramirez C. Modelo de miedo-evitación del dolor: evolución y nuevas propuestas. Rev Psicol la Salud. 2013;1(1):102-30.

12. Keefer L, Mandal S. The potential role of behavioral therapies in the management of centrally mediated abdominal pain. Neurogastroenterol Motil. 2015;27(3):313-23.

13. Sahota S, Fortun P. Disorders of gut-brain interaction and the long-term risks of opioids. Br J Hosp Med. 2019;80(10):C150-4.

14. Drossman DA, Tack J, Ford AC, Szigethy E, Törnblom H, van Oudenhove L. Neuromodulators for functional gastrointestinal disorders (disorders of gut-brain interaction): A Rome Foundation Working Team Report. Gastroenterology. 2018;154(4):1140-1171.e1.

15. Törnblom H, Drossman DA. Psychotropics, antidepressants, and visceral analgesics in functional gastrointestinal disorders. Curr Gastroenterol Rep. 2018;20(12):58.

16. Bharucha AE, Chakraborty S, Sletten CD. Common functional gastroenterologic disorders associated with abdominal pain. Mayo Clin Proc. 2016;91(8):1118-32. 